DOI: $10.25100 /$ pfilosofica.v0i51.10289

\title{
LOS FUNDAMENTOS ONTO-TEOLÓGICOS DE LA FILOSOFÍA POLÍTICA DE ALBERTO MAGNO
}

\section{José Ricardo Pierpauli}

Universidad de Buenos Aires. Consejo Nacional de Investigaciones

Científicas y Técnicas, Buenos Aires, Argentina.

\begin{abstract}
Resumen
El ideal propuesto por Santo Domingo de Guzmán a la Orden de Predicadores alcanzó, en la obra de Alberto Magno, su primera expresión académica y sistemática. La vita activa constituye una efusión de la vita contemplativa en la temporalidad. Ambas conforman lo que en la tradición dominicana recibe el nombre de vita apostolica. Objeto del presente estudio es mostrar que la contemplación no solo orienta el obrar moral y político, sino que fundamentalmente lo transforma. Los hombres no son buenos solo porque pueden ofrecer una explicación racional acerca de Dios como causa primera, sino porque, contemplando al Dios Revelado y Sus obras, se deciden resueltamente a traducir Su modelo en la vida política, mediante la virtus politica.
\end{abstract}

Palabras clave: orden politico; vida contemplativa; vida activa; vida apostólica; virtud política.

Cómo citar este artículo: Pierpauli, J. R. (2020). Los fundamentos onto-teológicos de la Filosofía Política de Alberto Magno. Praxis Filosófica, (51), 31-54. doi: 10.25100/pfilosofica.v0i51.10289

Recibido: 13 de febrero de 2019. Aprobado: 8 de abril de 2019. 


\title{
The Onto-Theological Foundations of Political Philosophy by Alberto Magno
}

\author{
José Ricardo Pierpauli ${ }^{1}$
}

\begin{abstract}
The thought proposed by St. Domingo de Guzmán to the Preachers Order has reached, in the work of Albert the Great, its first academie and systematic expresion. The vita activa is an effusion of the vita contemplativa in the temporality. Both of them constitute what, in dominican tradition, is called vita apostolica. The aim of the present paper is to show that contemplation not only guides moral and political action, but it basically transforms it. Men are not only good because they can offer a rational answer about God as a first cause, but because by contemplating the Revealed God and His Work, they decide to translate His model in political life, through the virtus politica.
\end{abstract}

Keywords: political order; contemplative life; active life; apostolic life; political virtue.

${ }^{1}$ Doctor en filosofía por la Universidad Nacional de Cuyo, Argentina. Profesor ordinario de Teoría del Estado en la facultad de derecho de la Universidad de Buenos Aires. Investigador indendiente de CONICET. Exbecario de CONICET, DAAD (Alemania), CNR (Roma, Italia). Conferencista en la Universidad de Hannover, Universidad de Frankfurt, Universidad de Bonn-Universidad de Roma La Sapienza. Área de especialización: Filosofía política e historia de la filosofía, especialmente la Edad Media y Edad Antigua.

ORCID: 0000-0003-1648-2350 E-mail: josericardopierpauli@yahoo.com.ar 


\title{
LOS FUNDAMENTOS ONTO-TEOLÓGICOS DE LA FILOSOFÍA POLÍTICA DE ALBERTO MAGNO
}

\author{
José Ricardo Pierpauli \\ Universidad de Buenos Aires. Consejo Nacional de Investigaciones \\ Científicas y Técnicas, Buenos Aires, Argentina.
}

I. El lugar que ocupa la Filosofía Política en el cuadro del saber articulado por Alberto Magno. La primera recapitulación del conocimiento humano a través de la Metafísica

En el horizonte de la via antiquorum en el que se inscribe la Filosofía Política de Alberto Magno, esta misma Filosofía se articuló como un todo de orden virtual. Por ello, el rigor sistemático de esa Filosofía Política es debido a sus relaciones de fundamentación recibida de los saberes subordinantes, que son la Teología Sobrenatural, la Metafísica del Ser y la hoy llamada Antropología Filosófica. La Filosofía Política de Alberto se subordina respectivamente al Bien Común Político, como analogado del Bien en sí y finalmente al Bien y a Dios, objeto de la Teología Sobrenatural. Pero, esa Filosofía es completamente incomprensible sin una previa reflexión acerca, en primer lugar, de la idea que se tenga acerca del hombre y, en segundo lugar, del obrar humano. La Filosofía Política de Alberto Magno es en rigor, $\theta \varepsilon \omega \rho i ́ \alpha$-theoria en su significación griega. La misma se traduce como contemplatio y, en esa perspectiva, posee dos orientaciones. La primera es horizontal y luego ascendente. La segunda es vertical y posteriormente descendente. Vale decir, una orientación horizontal que se dirige a las esencias y que es el comienzo de todo conocimiento ${ }^{2}$, para luego ascender hasta los últimos fundamentos

${ }^{2}$ Véase (Benavidez, 2018, p. 175). 
de esas esencias, y otra, vertical que desciende desde aquellos últimos fundamentos, hacia lo real concreto. Dos modelos podrían ilustrar lo dicho, a saber, el caso de Platón y el de Aristóteles. Platón, en la Alegoría de la Caverna, propone el ascenso purificador, vale decir, el camino filosófico de la ascesis. Aristóteles, por su parte, en su Libro X de la Ética a Nicómaco, ofrece un concepto de felicidad, tan vinculado con la Filosofia Práctica, como con la Especulativa. Respecto de la primera distinción; en la orientación horizontal, esa contemplación es, en primer lugar, Gnoseología-conocimiento- del objeto concreto. Posteriormente se hace scientia ${ }^{3}$, como conocimiento humano, universal y necesario. En su momento horizontal, la scientia se apoya en el

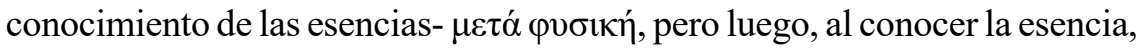
ese movimiento se torna vertical ascendente, para, consolidarse primero como sicientia en sentido riguroso. Luego, al ponerse frente a la majestad de Dios y explicar en esa amorosa presencia, los conocimientos adquiridos por la inteligencia, se torna auténtica sabiduría. Bajo el nombre de бopía

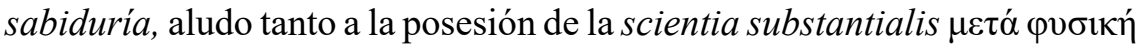
como a la suprasubstantialis- Teología Sobrenatural.

Ahora bien; ¿a qué llamo primera recapitulación del saber humano? Esa primera recapitulación tiene lugar cuando el conocimiento del objeto concreto queda cualitativamente transformado mediante la diafanidad de la luz de la Verdad del Ser. Así pues, el conocimiento político adquiere una nueva significación en el marco de su relectura desde la Metafísica del Ser y del Bien. El primer nivel de la scientia corresponde a una sabiduría rigurosamente humana, llamada por Alberto, scientia substantialis ${ }^{4}$ (Metafísica). El segundo nivel corresponde a lo que el Doctor universalis denomina scientia suprasubstantialis (Teología Sobrenatural) Se trata, en definitiva, de la articulación del conocimiento humano, en este caso, filosófico-político, en el más elevado nivel racional de la $\theta \varepsilon \omega p i ́ \alpha . ~ L a ~ \theta \varepsilon \omega \rho i ́ \alpha$ política recibe, según cuanto tiene de scientia, los resultados obtenidos de la contemplación. Luego, la $\theta \varepsilon \omega \rho i ́ \alpha$ Política es una scientia particular, la más importante en el orden práctico, que fluye de la $\theta \varepsilon \omega \rho i ́ \alpha$ en su más elevada significación ${ }^{5}$.

La sustancia ${ }^{6}$-ovбía- que es objeto de estudio en el nivel ascendente, es examinada por Alberto de dos modos. El primer modo; como participación

${ }^{3}$ Véase (Albertus Magnus, 2015, pp. 14-42). Véase también (Albertus Magnus, 1960, pp. 577-640).

${ }^{4}$ Véase (Albertus Magnus, 1972, pp. 9-58).

${ }^{5}$ A los fines de clarificar completamente la auténtica posición de la Orden de los Predicadores (Folghera, 1925).

${ }^{6}$ (Albertus Magnus, 1960, p. 60) 
de un Dios, tan solo considerado como causa primera. El segundo modo; según que la tesis de la participación se traduce como Creación. La sustancia es creada por Dios. La naturaleza y el hombre son sustancias creadas por Dios. Posteriormente, Alberto recibe los conceptos, que le ofrece la Física de Aristóteles, a fin de explicar el origen y la moción teleológica de las cosas creadas, conforme a una primera causa in-causada. Así, todo hombre es creado por Dios y se mueve según las mociones de un fin. La cualificación moral-política de tal fin es, visto desde la Filosofía Política, el Bien Común Político. Desde el punto de vista de este primer modo de considerar las sustancias-en el ascenso hacia el Ser- la $\theta \varepsilon \omega \rho i ́ \alpha$ se despliega más como contemplación, que como efusión práctica. Veámoslo con mayor claridad en la Figura 1. Ofrezco a continuación, una sistematización del saber ofrecida por Alberto, más allá de sus comentarios a la Filosofía Práctica de Aristóteles. Dicha sistematización fue articulada por el Doctor universalis en dos momentos a saber, antes de iniciar su labor como comentador de las obras de Aristóteles, y en 1262, cerca del final de esa tarea e incluso, de su labor científica.

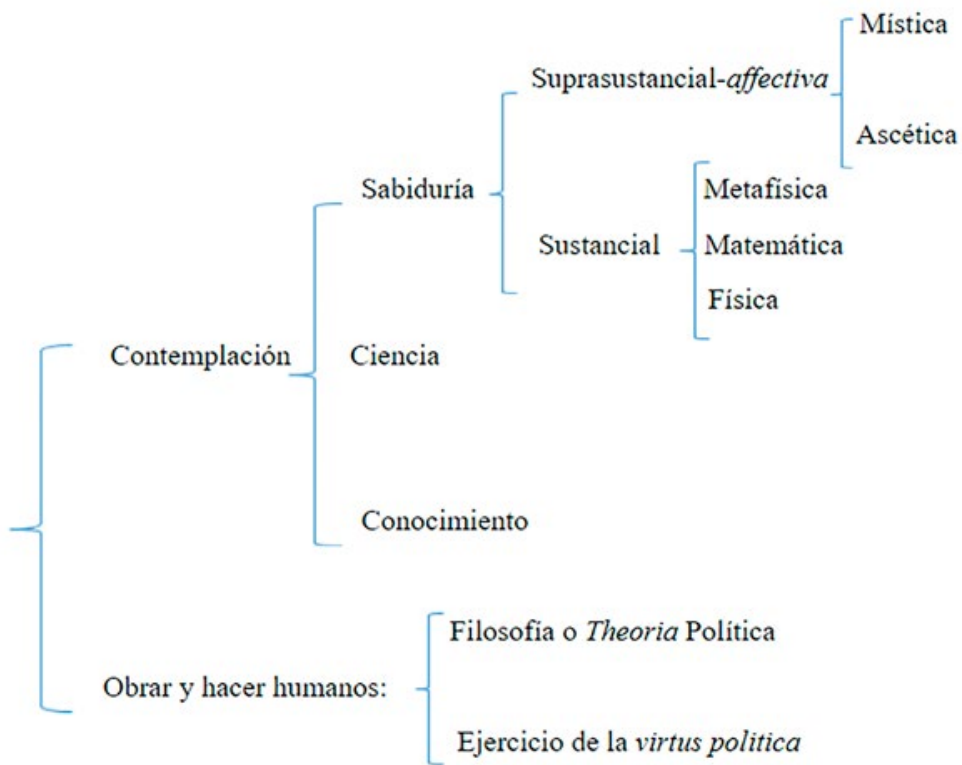

Figura 1. Primera Clasificación del saber en la obra de Alberto Magno

Dicha sistematización fue articulada por el Doctor Universalis en dos momentos a saber, antes de iniciar su labor como comentador de las obras 
de Aristóteles, y en 1262, cerca del final de esa tarea e incluso, de su labor científica ${ }^{7}$.

La segunda clasificación del saber, recogida de una obra posterior como es el segundo comentario a la Ética a Nicómaco (E-II de 1262) reviste particular relevancia. A la luz de ambas clasificaciones del saber, resulta claro que el Doctor universalis jamás concibió, ni la Ética, ni la Política, como saberes radicalmente autónomos de los saberes especulativos superiores a

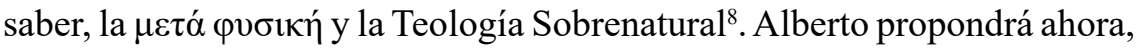
en sus años de plenitud filosófica y teológica, una clasificación del saber según la materia-de re philosophica. Y según el modo-de modo-de tratar la materia que es objeto de estudio. En esta segunda división se examinan los saberes, según sus respectivos objetos formales. Es importante destacar, que toda vez que hablamos de objeto formal, hablamos también de participación$\mu \varepsilon \dot{\theta} \theta \varepsilon \xi 1 \varsigma$ - metafísica. El Ser, como primer analogante, es Uno, que se participa en grados descendientes. Por ello, el saber acerca de esas modalidades del ser, el saber físico, el moral-político, se diferencia según los respectivos objetos formales de estudio. En este caso, tales objetos formales son la Física, la Moral y/o la Política. Obsérvese la Figura 2.

${ }^{7}$ Análogamente a lo dicho a propósito de la clasificación de las ciencias llamadas sustanciales, las prácticas fueron clasificadas por Alberto en el Prólogo de su Comentario Super ethica (E-I) (Albertus Magnus, 1975, pp. 3-54)

${ }^{8}$ A propósito del debate entre autonomía y subordinación/subalternación de la Ética y de la Política conviene completar las presentes reflexiones con las vertidas por Jorge Aurelio Díaz (2017). 


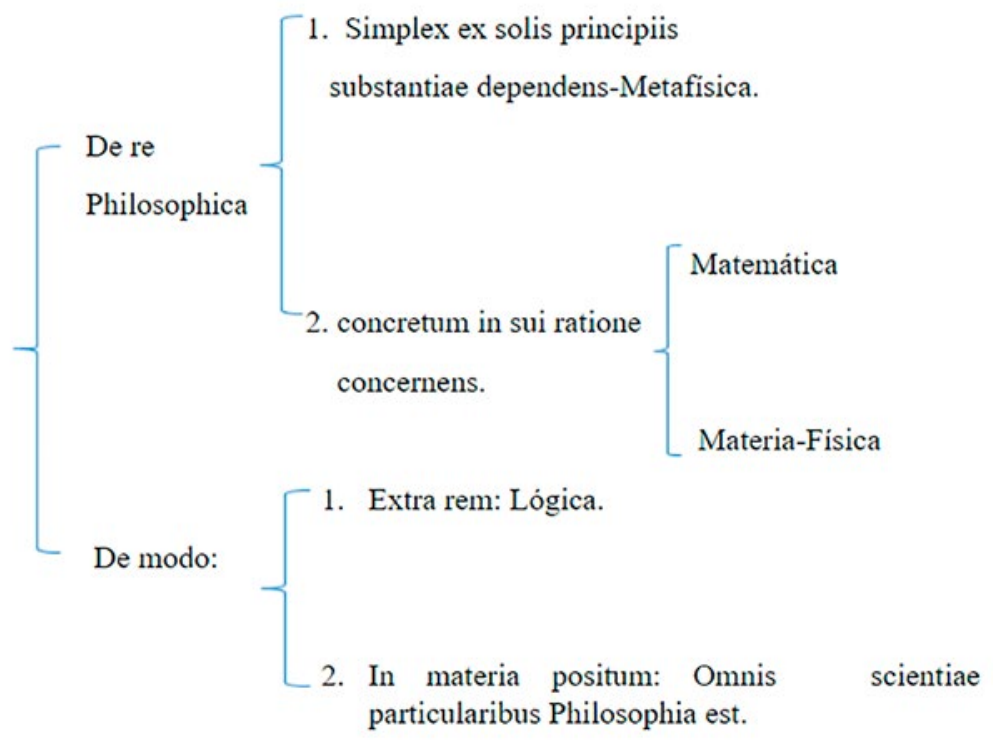

Figura 2. Segunda clasificación del Saber en la obra de Alberto Magno

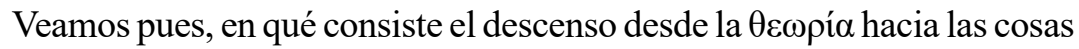
del orden práctico-moral y político. Dado que la persona humana es sustancia racional, el Doctor universalis examina y expone, luego de considerar la moción física del hombre en vistas de un fin y su justificación metafísica y teológica, aquellas mociones más específicas, que tienen lugar, según una nueva moción, esta vez moral-política, fundada en la misma naturaleza. Parece que todos los hombres así como los grupos políticos persiguen un cierto bien (Aristóteles ${ }^{9}$ ) Esta segunda moción, recibe su orientación primeramente, desde el propio Bien Común Político, y, posteriormente, del recto gobierno, que la razón ejerce sobre los afectos y sobre la voluntad. Mediante el primer movimiento de ascenso y en este segundo de descenso, puede operarse la primera recapitulación del conocimiento humano, según la perspectiva filosófica, que acerca del Ser y del Bien puede alcanzar el hombre, apoyado tan solo en la luz natural de la razón. No es lo mismo poseer experiencia política, que conocer el suceder de esa experiencia por las causas universales y necesarias. La mera $\delta$ ó $\xi \alpha$ acerca de cuestiones

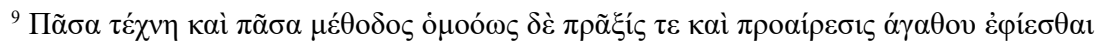

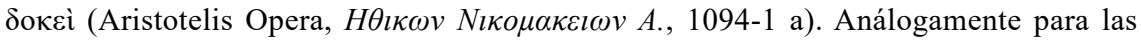

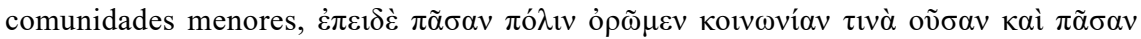

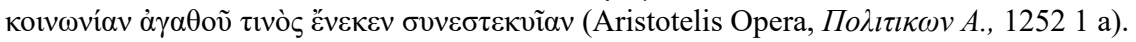


políticas, o mejor aún, el conocimiento primario, queda cualitativamente transformado a la luz de la Metafísica. Hasta aquí, el ideal político del mundo griego que Alberto comenta.

\section{La segunda recapitulación del conocimiento humano}

La primera recapitulación del conocimiento humano, la que podríamos denominar recapitulación metafísica, nos permiten experimentar ahora lo que podemos llamar una segunda recapitulación, vale decir, una recapitulación teológica, del conocimiento humano. Ello tiene lugar cuando el hombre contempla a Dios, ya no apenas como Causa Primera del orden natural, sino a partir del conocimiento de Dios, que nos entregan la Teología Sobrenatural y la Fe Sobrenatural. Vale decir, la scientia suprasustantialis y la Fe Religiosa.

Esta segunda recapitulación del saber humano puede ofrecer al conocimiento filosófico-político una transformación cualitativa aún más profunda, que la primera, si es que, más allá de la Metafísica, opera en nosotros una cierta Revelación del Deus absconditus ${ }^{10}$. Mientras la primera recapitulación se cumple a través de los tres grados de abstracción-FísicaMatemática y Metafísica- esta segunda recapitulación del conocimiento puede operarse según tres nuevos grados de perfección, a saber, mediante la sola aceptación de la Fe Sobrenatural, mediante la aceptación de la Fe Sobrenatural y el cultivo de la Teología como Scientia divina, y, por último, en el grado más elevado y perfecto, mediante la experiencia mística. Obsérvese la Figura 3.

1. Primera recapitulación: Desde el simple conocimiento a la Metafísica del Ser y del Bien mediante la abstracción física, la matemática y la metafisica

2. Segunda recapitulación; del simple conocimiento a:

A. Fe Sobrenatural.

B. Cultivo de la scientia divina.

C. Experiencia mistica

Figura 3. Las dos recapitulaciones del Conocimiento humano

${ }^{10}$ Véase (Anzulewicz, 2003, p. 169). 
Ciertamente, este último grado de perfección del saber humano, el de la experiencia mística, queda reservado a pocos. La unión mística con el Deus absconditus es la más elevada cualificación del alma, la coronación sublime del camino de ascenso-sea filosófico-teorético y/o práctico, y la propedéutica espiritual de la óptima vida moral y política. Se trata, en este último nivel, de la sabiduría auténtica, o si se prefiere, del conocimiento a la luz de la Divinidad. Aquí sabiduría conlleva para Alberto, el sabor de las cosas divinas, que se experimenta mediante la experiencia de la Divina Sabiduría, del ejercicio de las virtudes allí fundadas y de la posesión de las bondades, que se obtienen como frutos ${ }^{11}$.

Si consideramos el esfuerzo de la inteligencia humana por conocer a Dios desde sí misma, se trata entonces de un cierto ascenso purificador, que Alberto sitúa como eje de la totalidad de las disciplinas filosóficas. La Filosofía de Alberto, obviamente incluida su Filosofía Política, es también un ascenso del espíritu orientado decididamente hacia Dios Creador, cuya más alta perfección tiene lugar cuando esa Filosofía puede traducirse como amor a Dios. Ese amor a Dios, es piedad personal, si consideramos al hombre solo, en cambio, se transforma en amor de Dios cuando su objeto es el prójimo, $\mathrm{y}$, de este modo, recibe el nombre de Caridad Sobrenatural. Por su parte, la Caridad es el fundamento de la amistad politica, que articula la Comunidad Política. Luego, ambos movimientos, el ascendente y el descendente, en la Epistemología de Alberto, no ofrecen tan solo un valor epistemológico e iluminador de la $\theta \varepsilon \omega \rho i ́ \alpha$ Política, sino estructurador y cualitativamente transformador de la misma.

Alberto considera la posibilidad de conocer a Dios, ya no partiendo solamente del ascenso, que la inteligencia humana puede llevar a cabo, sino en virtud de la acción positiva de Dios que, subsanando los límites de la inteligencia, nos acerca hacia E ${ }^{12}$. Por ello, una reconstrucción del camino del pensamiento filosófico de Alberto Magno requiere el emplazamiento de sus tesis filosófico-políticas, especialmente las recibidas y articuladas a partir de las tradiciones que le precedieron en la Antigüedad, en el marco de sus obras decididamente neo-platónicas, tales como los escritos a propósito del Corpus dionysiacum areopagiticum.

${ }^{11}$ Sapiens proprie dicitur, qui sapore divinorum et experimento divinae sapientiae, virtutis, et bonitates, accepit notitia. Is. XI-2: Requiescet super eum spiritus Domini, spiritus sapientiae, Albertus Magnus, Super Osee 14, 10, ed. París, T 19, p. 123 a, citado por (Fries, 1994, p. 24).

${ }^{12}$...ultra vis nostras est, sed cum ratio fide iuvatur, valet in id quod ante no valuit, unde Hebr. XI-1: Fides est substantia rerum sperandum, argumentum non apparentium, e II ad Cor III-5: Non quod suficientes simus cogitare aliquid a nobis quasi ex nobis, sed sufficientia nostra a deo est (Albertus Magnus, 2015, pp. 5-17). 


\section{Las condiciones previas y las consecuencias de las dos recapitulaciones del conocimiento filosófico-político. El cuarto grado de abstracción introducido por Alberto como intento de resolución del hiato gnoseológico entre el hombre y Dios}

La primera relectura del saber humano político exige, según Alberto, de ciertas condiciones previas. En efecto, por via resolutionis, ascendemos hasta los límites mismos de la inteligencia humana ${ }^{13}$. Aun cuando precisamente en ese límite solo encuentra el hombre al dios filosófico, vale decir al equivalente teológico de la primera causa aristotélica, ese ascenso requiere de una cierta pureza afectiva, espiritual e intelectual, que se cristaliza como obrar honesto. La misma se obtiene de tres modos. Desde el punto de vista especulativo, a través del cuarto grado de abstracción, desde el punto de vista práctico, mediante el ejercicio perseverante de la virtud política $\mathrm{y}$, desde el punto de vista de la piedad religiosa, a través de la apertura del alma a la Gracia Santificante. Lo dicho puede expresare también de este modo; el camino auténticamente filosófico, presupone, según Alberto, la honestidad intelectual y moral y la pureza de nuestros afectos. Recién, entonces, es posible emprender el vuelo hacia la scientia substantialis en sus dos acepciones, metafísica y teológica, que es en definitiva y en virtud de tales motivos, scientia glorificans et scientia affectiva. En cambio, en la dirección vertical descendente, esa contemplatio se torna acción concreta. En la obra de Alberto, como también en la obra del discípulo Tomás de Aquino, la sabiduría auténtica, la scientia substantialis-Metafísica- constituye el firme fundamento y criterio de legitimidad, tanto de la acción moral y política, como del quehacer estético ${ }^{14}$.

Así, por tanto, la segunda recapitulación del conocimiento presupone la Fe Sobrenatural, el cultivo de la Teología Sobrenatural y finalmente, la experiencia mística. Posteriormente, en el descenso filosófico, la virtus politica queda completamente transformada, según la luz superior de la Fe y de los Misterios del Deus absconditus ${ }^{15}$. De este modo, Alberto lleva hasta sus últimas consecuencias el neo-platonismo cristianizado, toda vez que transforma su Filosofía en un cierto ordo amoris Dei. Esta segunda disposición del espíritu constituye el punto de partida y la conditio sine qua non de la vita apostolica. Ese tipo de vida está hoy, como antes, solo

${ }^{13}$..quia nos non possumus congnoscere causam ipsam, secundum quod est simplex in se, ut eam accipiamus cognitionem causati, sed potius e converso, ideo est alius modus cognoscitivus, quo per causata accipiamus causam; et hic est resolutorius, et talis est modus huius scientiae (Albertus Magnus, 1972, pp. 3-6).

${ }^{14}$ Véase también (Sancti Thomae Aquinatis, 1888, p. 12).

${ }^{15}$ Vere tu es deus absconditus, deus Israel, salvator, Is. XLV-15 (Albertus Magnus, 1978a, pp. 453-454). 
reservado a quienes, en la opinión de Joseph Ratzinger, constituyen las elites intelectuales y politicas, o bien de las llamadas elites creativas. ${ }^{16}$

\section{A. El cuarto grado de abstracción y la insuficiencia de la Filosofía}

Me propongo abordar aquí, el siguiente problema: a saber, el de la novedad introducida por Alberto Magno en punto al camino de la reflexión filosófica, mediante la inclusión de un cuarto grado de abstracción. He aquí la clave que posibilitará comprender la transformación del ordo politicus en ordo amoris. Me interesa subrayar la insuficiencia de la Filosofía en términos de conocimiento, cuando el espíritu humano se encamina nada menos que hacia el conocimiento de Dios. Dado que el cuarto grado de abstracción fue definido por Alberto con referencia al Dios Revelado, entonces ese camino del espíritu es el que permitirá superar, de un modo ya no sólo filosófico, lo que he definido como la insuficiencia de la Filosofía ¿Puede seguirse el conocimiento de Dios luego y más allá del conocimiento político al que aspira, por ejemplo, una $\theta \varepsilon \omega \rho i ́ \alpha$ del Estado? Resueltamente, sí, pues el ascenso hacia ese altísimo conocimiento constituye, al menos como moción fundamental, el natural movimiento del espíritu de un homo politicus que, mediante ese movimiento del espíritu, se torna posteriormente homo religiosus.

El cuarto grado de abstracción introducido por Alberto, a saber, el de la transformación afectiva del alma humana en vistas y a partir del amor de Dios, da por concluida la pura ascesis filosófica, que se logra mediante la primera recapitulación del saber humano. Esa primera recapitulación, que se recorre mediante los conocidos tres grados de abstracción, nos ofrecía apenas una relectura metafísica del conocimiento humano. En cambio, con la transformación del alma humana, comenzamos a recorrer el camino de ascenso y aun de unión con Dios que dará más tarde como resultado, aquello que he llamado la segunda recapitulación del saber humano. Bien puede postularse que ahora, en el marco de esta segunda recapitulación, ya no se trata simplemente de saber filosóficamente acerca de las cuestiones políticas, sino de saber esas mismas cuestiones según la luz superior de la Fe, de la Sagrada Teología y, en síntesis, de obrar en consecuencia según el amor de Dios en la comunidad política.

${ }^{16}$ La exigencia del cultivo del intelecto y de la sabiduría por parte del gobernante aparece claramente sugerida por Alberto Magno en el mismo punto de partida de su Prólogo al Comentario a la Política de Aristóteles. En cambio, esa misma tesis, a saber, la del político ilustrado fue recientemente puesta en boga por Joseph Ratzinger mediante su idea de elites creativas y/o intelectuales transformadoras del orden político y moral. Véase (Granados, 2017) 
Por cierto, la Filosofía por sí sola no acaba de modo inmediato en el conocimiento esencial de Dios Revelado. Por ello, debe postularse la existencia de un cierto hiato gnoseológico entre el hombre y Dios. En este cuarto grado de abstracción lo que permite superar el conocimiento racional de Dios, es, según Alberto, la esfera afectiva del alma humana. El amor a Dios es para Alberto, un nuevo modo de conocer, que transforma el alma en su totalidad. Desde el punto de vista del predominio de estos afectos, ahora sublimados, el examen del cuarto grado de abstracción es filosófico, pero según que trata acerca de los afectos como potencia del alma, es más extra y supra filosófico, es decir, pertenece a la Teología Sobrenatural, según que tales afectos tienen a Dios por objeto. Así, por tanto, el cuarto grado de abstracción constituye un concepto limítrofe, vale decir, en parte filosófico y en parte teológico. Cabe a la Teología Sobrenatural una reflexión acerca del amor a Dios en la medida en que ese amor glorifica el alma humana y la eleva.

Ahora bien, según que el cuarto grado de abstracción glorifica el alma y la transforma, toca a la Filosofía Práctica verificar su incidencia en lo que hemos definido como $\theta \varepsilon \omega \rho i ́ \alpha$ Política. Una vez experimentada la transformación glorificante del alma, estamos pues en condiciones de operar la segunda recapitulación del saber acerca de la Política. A partir de allí, la virtud política, que es el eje, el cardan de la completa vida del hombre ${ }^{17}$, se ejerce no sólo por amor al Bien Común Político, sino fundamentalmente por amor a Dios, que es el primum analogans de ese Bien. Alberto ha resuelto, mediante el recurso a las potencias afectivas del alma, aquel deseo natural por conocer a Dios y para el cual, todo saber humano se declara insuficiente. De este modo, el Doctor universalis pudo ampliar y transformar el horizonte gnoseológico trazado por Aristóteles en el punto de partida de su $\pi \varepsilon \rho i ́$ $\psi v \chi \eta \varsigma^{18}$ No obstante, ¿el amor a Dios resuelve por completo el problema de la distancia cualitativa e infinita que media entre Dios y los hombres? O bien ¿hay otro modo posible de resolver ese hiato gnoseológico?

\section{B. Tentativa de resolución de una objeción a propósito de los límites del conocimiento humano filosófico para conocer a Dios}

Intentaré resolver ahora una posible objeción, a propósito de la necesidad de conocer a Dios y de las limitaciones del intelecto humano para alcanzarlo. En efecto, es importante puntualizar que Alberto, en su Super ethica (E-I) señala que es imposible dirigirse a un fin determinado sin conocer

\footnotetext{
${ }^{17}$ Véase (Albertus Magnus, 1974, pp. 37-8).

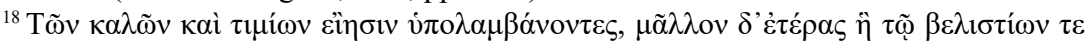

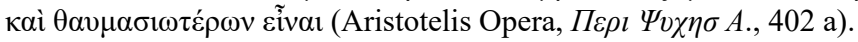


previamente ese fin ${ }^{19}$. Pero, si la reflexión medieval que los autores, como Alberto, heredaron de los Padres de la Iglesia se ordenaba, no a la mera erudición, sino a la salvación de las almas ${ }^{20}$, entonces el camino filosófico -quia est-resulta claramente insuficiente, aunque necesario a los ojos del Doctor universalis, pues le está vedado al hombre conocer a Dios tal como es. Luego, la exigencia de conocer el fin, no sería aplicable al conocimiento de Dios, por lo demás según dije, un conocimiento absolutamente indispensable para consumar la intención general del pensamiento del Doctor universalis, a saber, la configuración de la vida apostólica en el ordo amoris que es la vida política comunitaria.

¿Por qué fracasan la Filosofía y su Teología Natural en la tentativa de conocer a Dios? Al hombre le está reservado, si es que recurre tan solo a la razón natural, un conocimiento de Dios en términos de primera causa y de moción universal del todo de la Creación. Cabe reiterarlo, el hombre solo puede conocer a Dios quia est ${ }^{21}$ pero no quid est ${ }^{22}$. Conocer a Dios como causa primera presupone conocerlo-via resolutionis- por el camino de los efectos hacia las causas ${ }^{23}$. No obstante las limitaciones de la pura racionalidad, Alberto propone alcanzar finalmente un conocimiento del Dios Revelado, pues ese tipo de conocimiento, aunque vedado al hombre bajo ciertos límites, es absolutamente necesario, a fin de alcanzar, por la vía ascética y mística, el conocimiento de Dios que, en la perspectiva de Alberto, aun antes de abrirle el camino de la salvación eterna, purifica la inteligencia y la transforma, ya en esta vida, en vistas del obrar humano en obrar virtuoso sub specie aeternitatis ${ }^{24}$. Naturalmente, debido a que la

${ }^{19}$ Véase (Albertus Magnus, 1975, pp. 7-10; Sancti Thomae Aquinatis, 1888, p. 6.)

${ }^{20}$ Véase (Geyer, 1967, p. 3 y ss.)

${ }^{21}$ Véase (Albertus Magnus, 1972, pp. 10-64 ; 11-36 y 32-14).

${ }^{22}$ Véase (Albertus Magnus, 1972, pp. 2-55, 11-16, 13-38 y 32-14).

${ }^{23}$...quia nos non possumus congoscere causam ipsam, secundum quod est simplex in se, ut per eam accipiamus congintionem causati, sed potius e converso, ideo est alius modus cognoscitivus, quo per causata accipiamus causam; et hic est resolutorius, et talis est modus huius scientiae, non secundum quod fit resolutio in causam, prout est ignota propter eminentia sui, ad modum significandi per nomen, quia hoc pertinet ad mysticam theologiam, sed secundum quod fit resolutio in causam, prouet est univoce producens causatum (Albertus magnus, 1972, pp. 3-5).

${ }^{24}$....sicut dicit Apuleius ad libro De deo socratis, quod deus vix a sapientibus viris, cum se vigore animi, in quantum licuerit, a corpore removerint, velut in altissimis tenebris candidum lumen intermicat (Albertus Magnus, 1972, pp. 7-31). Alberto cita aquí, como en otros lugares de su obra a Apuleyo. Se trata de un sofgista de orientación platónica que vivió durante el siglo 2 AC. Durante su vida gozó de gran prestigio, fundamentalmente en su defensa contra las acusaciones de ser promotor de la magia. Parte de sus escritos fueron conocidos en la Antigüedad tardía. En la Edad Media alcanzaron fama las siguientes obras de Apuleyo: Metamorphoses, Florida Apologia pro se de Magia y, claro está, la obra que 
amorosa unión mística es unión misteriosa, no es completamente exigible al hombre político virtuoso. A este, cuando menos, se le requiere fundar su vida moral y política en un Dios Creador cognoscible a través del orden natural y de las explícitas revelaciones del Creador en las Sagradas Escrituras. La Fe Católica del hombre individualmente considerado tiene, como era el caso del contemplativo y del místico, una clara orientación política. Aquí como en el caso anterior, se verifica también la idea de un obrar moral y político como momentos de la contemplación. El hombre político, aunque inmerso radicalmente en la temporalidad, no deja de contemplar a Dios en el horizonte de su obrar cotidiano. La vida política virtuosa es también una efusión de la Fe Sobrenatural ${ }^{25}$.

Pues bien, se mira por el género humano, cuando también a los aún no capaces del conocimiento de las realidades espirituales y eternas los nutre la fe en la historia temporal que, después de los patriarcas y profetas, por nuestra salvación dirigió, precisamente por el sacramento del hombre asumido, la excelentísima fuerza y sabiduría de Dios-1 Cor. 1-24- en la cual está la salvación para todo el que cree-Rom. 1-16- a fin de que cada uno, impulsado por la autoridad, esté al servicio de los preceptos, purificado por los cuales y enraizado y fundamentado en la caridad -Ef. 3-17- pueda, no ya pequeñín de leche, sino joven que come, correr con los santos, comprender la anchura, largura y profundidad, conocer también el supereminente conocimiento de la caridad de Cristo-Ef. 3-18/19-26

Como queda claro, la intención de Alberto trasciende los límites del aristotelismo típicamente griego y específicamente árabe, que como se sabe, hizo de Dios, por lo menos en el caso de Alfarabi, apenas un dios cosmológico. La referencia al Dios de Israel constituye, en definitiva, el $\alpha$ y el $\omega$ de la vida política. El Bien Común Político es el Bien querido por el Dios Único, un bien para el aquí y el ahora que el alma del gobernante justo y sabio, orientado hacia Dios, debe y puede discernir en el aqui y en el ahora, mediante el ejercicio de la virtud de la Prudencia Política Arquitectónica.

\footnotetext{
aquí parece haber utilizado Alberto, a saber, Deo Socratis. Además se cuenta entre sus obras filosóficas De Platone et eius dogmate (Hellmann, 2002, pp. 818-819).

${ }^{25}$ Para verificar la tesis que se opone a la que aquí ofrezco, conviene leer la argumentación recientemente ofrecida por los siguientes autores: Véase (Müller, 2006, p. 554; Honnefelder, 2011, pp. 21-22; Höffe, 1996b, pp. 185 y ss; Höffe, 1996a, pp. 56 y ss).

${ }^{26}$ Véase (Agustín de Hipona, 2015).
} 


\section{El camino aristotélico emprendido por Alberto Magno y los aportes de las doctrinas de Euclides}

Alberto es un aristotélico consecuente con los ideales de su orden. Por ello, parte del reconocimiento del camino que va desde los efectos a las causas. Sin embargo, en ese mismo camino resolutivo, no es solamente aristotélico, sino que toma también dos modelos, uno de la perspectiva y de la óptica de Euclides y el otro de la Geometría, para demostrar la existencia de un principio absoluto y al mismo tiempo, la imposibilidad de conocerlo completamente, aunque sí de modo relativo. A propósito de la óptica de Euclides, señala que, humanamente, podemos ver la realidad de Dios, pero se trata de un ver análogo al que es propio de la óptica, esto es, desde un determinado ángulo de mira, es decir, desde el ángulo de mira del observador. En efecto, desde allí recorremos de un modo limitado, por lo demás, propio del ojo humano, una realidad compleja como la de Dios. Luego, tan solo podemos observar la realidad de Dios, según una parte de la misma, que coincide con el ángulo de nuestra perspectiva óptica. Tan solo esa es la medida en que Dios se nos pone delante de los ojos, y por tanto, permite que por ese medio sensitivo, lo conozcamos ${ }^{27}$. En cuanto al modelo geométrico, Alberto afirma que, siendo la semirrecta una línea compuesta por la unión de puntos que, partiendo desde un punto fijo, se proyecta hacia el infinito, la misma puede ser conocida por nosotros, aunque solo conozcamos el punto de origen, a pesar que no podamos conocer la infinitud de los puntos que la constituyen. Así mismo, siendo Dios el principio primero de la infinita Bondad, podemos conocerlo precisamente como punto de partida de toda Bondad, de Toda Perfección en suma, Omnipotente, Omnisciente y Omnipresente, sin que sea necesario, conocer con los ojos humanos, la infinitud de tales determinaciones acerca de $\operatorname{Dios}^{28}$.

\section{Vita activa como efusión de la vita contemplativa. Hacia la configuración del ideal apostólico dominicano como vita apostolica} No obstante, el acenso filosófico, a cuyo resultado he designado como primera recapitulación del saber humano, no puede resolver por sí solo, el hiato gnoseológico que lo separa de la infinitud y de la complejidad de Dios pues, aun el camino resolutivo al que hice referencia a propósito del cuarto grado de abstracción, es todavía insuficiente. Lo es porque, por vía resolutiva, podemos precisamente resolver en uno, las partes de un todo compuesto. La vía resolutiva comienza por la realidad tangible como aquella que es hoy para nosotros objeto de una Teoría del Estado, para elevarse a

\footnotetext{
${ }^{27}$ Véase (Albertus Magnus, 1972, pp. 10-77).
}

${ }^{28}$ Vése (Albertus Magnus, 1972, pp. 15-25). 
las realidades inteligibles. Ese camino interrumpe su ascenso frente a la realidad de un Dios, que sobrepasa ilimitadamente la inteligencia humana ${ }^{29}$. Se trata del punto de llegada, el más elevado a que pueden aspirar las ciencias substanciales. Ese camino, mirado en retrospectiva, reconoce tres tramos, que coinciden con los tres grados de abstracción. Sin embargo, la Filosofía, que se apoya solo en los tres conocidos grados de abstracción fracasa, si su propósito es conocer al Dios Revelado, Quien es en última instancia, el fundamento inconmovible de la vida práctica. La Filosofía, según Alberto, no puede entregarle al alma, más que limitadamente, el reposo contemplativo pues, una vez agotados sus caminos, el conocimiento imperfecto y necesario de Dios hacia el que, a pesar de esta insuficiencia, la inteligencia humana no renuncia, requiere ahora, para resolver este hiato gnoseológico, del concurso efectivo de Dios mismo ${ }^{30}$. Uno de los modos en que ese concurso Divino se torna realidad es la transformación del alma humana, que se obtiene mediante el cuarto grado de abstracción.

La tesis precedente nos aproximó hacia la resolución del problema del modo en que, por la vía ascética, el hombre puede, a pesar de sus propios límites, abrirse al misterio de Dios. La idea que recojo de Alberto a continuación, permitirá comprobar de qué modo resuelve ese hiato que separa la inteligencia humana de Dios, apoyándose en su Teología Mística. En efecto, el Doctor universalis enuncia los tres grados de abstracción más conocidos, y mediante los cuales podemos filosofar acerca de nuestros objetos de conocimiento. Ellos son, en primero lugar, el nivel de abstracción Física. El segundo es el grado de abstracción que se opera mediante el recurso a la Matemática y, finalmente el tercero, es el más elevado y corresponde a la llamada Filosofía Primera o Metafísica. Esos tres grados de abstracción indican una progresiva profundización en el conocimiento de un determinado objeto, en el lenguaje aristotélico que Alberto recibe, el ascenso a través de

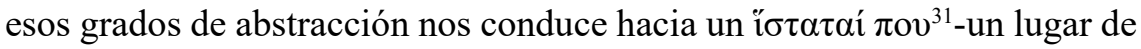

${ }^{29}$...mens non potest uniri deo secundum totum ambitum suae infinitatis, ita scilicet quod cognoscat ipsum et nihil de ipso sit extra ipsam, quia sic comprehenderet ipsum; potest tamen uniri sibi, inquantum est obiectm eius, attingendo ad ipsum secundum rationem, secundum quam sibi obiectum est (Albertus Magnus, 1972, pp. 33-32)

${ }^{30}$...scientia, quae procedit ex principiis rationis, ponit in aperto ea ad quae ducit. Huiusmodi autem doctrina no procedit ex talibus principiis, sed potius ex quodam lumine divino, quod non est enuntiatio, per quam aliquid affirmetur, sed res quaedam convincens intellectum, ut sibi super omnia adhaereatur. Et ideo elevat intellectum ad id quod excedit ipsum, propter quod remanet intelelctus in quodam non determinate noto. El hoc lumine proportionatur lumini, quo corporalis visus confortatur ad videndum, quod tamen non facit alicuius visibilis determinatam cognitionem (Albertus Magnus, 1978, pp. 455-12).

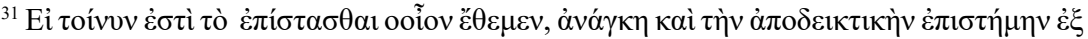

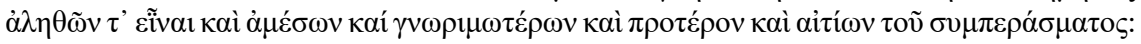


reposo-que es, según se verá un $\alpha \rho \chi \eta ́$-principio- $\kappa \alpha \theta^{\prime} \varepsilon \xi o \chi \eta ́$-por excelencia. No obstante, Alberto pone su atención en el hecho, que el natural movimiento del espíritu no se detiene en el Ser, sino que indaga acerca de su último fundamento. Allí, donde el intelecto humano se encuentra con Dios, pone en evidencia, al mismo tiempo, la más acabada prueba de su insuficiencia para conocerlo. Es allí donde Dios atrae hacia si la totalidad del hombre, pero transformando los afectos con una fuerza irresistible. Ese último movimiento fue caracterizado por Alberto como el cuarto grado de abstracción, al que considera el más perfecto y preferible. Es entonces a partir de esa profunda transformación, que puede iniciarse la última y la más acabada relectura del saber y del obrar humano moral-político. Es a partir de allí, que podemos reformular una Filosofía Política a la que deberíamos llamar, ya no Filosofía, sino $\theta \varepsilon \omega \rho \iota \alpha$, o más específicamente, auténtica sabiduría Política ${ }^{32 .}$.

\section{E. La noción del Bien en el neo-platonismo de Alberto. Su integración con la teleología aristotélica}

La noción de Bien es primera en el reino de los fines, pero también lo es en el orden de los principios. Este matiz típicamente neo-platónico, el de la primacía del Bien sobre el Ser, resulta difícilmente cuestionable en Alberto, pues es evidente que Dios no hace cosa alguna, a no ser mediante un acto de amor infinito y de pura bondad ${ }^{33}$. En otras palabras, Alberto parece trasladar al plano filosófico la siguiente idea, si bien es rigurosamente cierto que el actus essendi de cada criatura depende, en cada tramo de su concreción temporal, del Ser Divino, no por ello debe pasarse por alto, que Dios confiere el ser ante todo, en virtud de su infinita bondad. Por ello, para el Doctor universalis, la virtud política, que se cualifica, según la perspectiva del Bien Común Político, es, ante todo, ordo amoris, amor por el bien de la

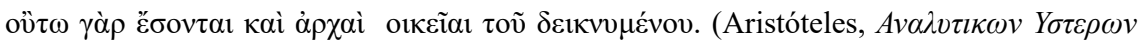
A., 71 B 20)

${ }^{32}$...sunt enim in nobis tres unitiones, quibus intellectus noster coniungitur cognoscibilibus, quae sunt philosophiae. Prima est secundum abstractionem universalis a particularibus, et haec est in naturalibus; alia est per maiorem abstractionem, scilicet formae a materia, secundum distinctivam rationem et veritatem essentiae in his quae tamen secundum esse non sunt a materia separata, et haec est in mathematicis, ponens supra sensum in ordinem imaginabilium; tertia est quantum ad ea quae secundum rem non dependent a sensibilibus, et haec sunt in ordine intelligibilium tantum, scilicet cum est consideratio de substantia et de principis eius, quae secundum quod huiusmodi non dependent a sensibilibus, et haec est in metaphysica....Sed quartum est melior, quae est per ipsum lumen divinum intelligibile uniens intelligibilibus, et de ista loquitur hic (Albertus Magnus, 1972, pp. 5-23).

${ }^{33} \mathrm{El}$ primer analogante de este Bien, no es específicamente el Bien que se estudia como trascendental del Ser, sino el Bien por excelencia que se identifica como el Espíritu Santo, fruto del Amor del Padre por el Hijo (Albertus Magnus, 1972, pp. 46-71). 
Comunidad Política. Solo en esta perspectiva el hombre se ordena al Estado y no este hacia aquél.

En virtud del razonamiento precedente, la Filosofía de Alberto tiende, por intrínseca necesidad, a tornarse Teología Sobrenatural. He aquí el segundo modo que dispone el alma para abrirse, no al mero conocimiento, sino al misterio de Dios. Se trata de un modo diferente de formular una verdad ya anticipada, a saber que la Filosofía de Alberto, la completa categorización de los saberes ofrecida por Alberto, incluida su Filosofía Política, no es otra cosa que un camino contemplativo y activo abierto a la Trascendencia. Resumidamente; el hiato gnoseológico al que hice referencia es el que separa el fin del camino filosófico, del comienzo de la unión con Dios. Ese hiato puede superarse parcialmente, recurriendo al cuarto nivel de abstracción y puede aún profundizarse más mediante la acción gratuita y misteriosa de Dios. Alberto hace valer una doctrina estoica, que parece recoger de Cicerón y que fuera explícitamente enunciada en el Prólogo de su Comentario Super ethica (E-I) En efecto, el fin, especialmente cuando nos referimos al hombre virtuoso, o a Dios, nos atrae hacia si mismo para que lo imitemos. Ello vale, no solo para el uso místico y ascético que le doy aquí, siguiendo a Alberto, sino para el uso moral y político, que el Doctor universalis hace de tal principio en los comentarios a la Ética a Nicómaco ${ }^{34}$. En la resolución del llamado hiato gnoseológico, Alberto anticipa aquella tesis de Tomás de Aquino en virtud de la cual el Aquinate definía la Filosofía como ancilla fidei. Tomás recoge esa impronta de su maestro también cuando, al comienzo de la Suma Teológica, nos hace ver la bondad de Dios, que revelándose al hombre, le facilita un tipo de conocimiento necesario, que el hombre, por sus propias limitaciones, solo alcanzaría al cabo de mucho tiempo y con mezcla de muchos errores ${ }^{35}$.

De este modo, mediante la inclusión del cuarto grado de abstracción, es como el Doctor universalis, rehabilita la inteligencia humana, o bien la totalidad del alma humana, tornándola capaz de tres cosas. La primera, de conocer a Dios, según que Dios mismo lo permite, mostrándosele al hombre in via según conviene, la segunda, le permite operar una transformación interior de tal profundidad que, en el camino que he designado como descenso filosófico, esto es, la traducción de la contemplatio en agere ${ }^{36}$,

${ }^{34}$ Véase (Albertus Magnus, 1987, pp. 1-48; 713-11).

${ }^{35}$ Véase (Sancti Thomae Aquinatis, 1888, p. 6)

${ }^{36}$... in felicitate contemplativa non accipitur tantum ut verum, ut est perfectio intellectus, sed etiam ut bonum ipsius, et sic ex coniunctione ipsius ad intellectum sequitur delectatio (Albertus Magnus, 1987, pp. 709-10). 
hace posible obrar políticamente en la perspectiva de la divinidad ${ }^{37}$. Por último, puede el hombre, completamente transformado por Dios, cumplir acabadamente el ideal de vida mixta que es vita apostolica. La última especificación de la vita apostolica es la virtud política. Dicho de otro modo, vivir según las virtudes políticas, de la Prudencia, la Justicia, la Templanza y la Fortaleza, iluminadas desde su interior por las virtudes dianoéticas o intelectuales, y por las virtudes sobrenaturales de la Fe, de la Esperanza y de la Caridad, es en concreto, una forma, tal vez la más elevada forma de ejercer el apostolado propuesto por Santo Domingo de Guzmán.

\section{F. El intelecto adquirido ${ }^{38}$ como resultado de las dos recapitulaciones del conocimiento humano}

A fin de poner en evidencia la intención trascedente de la Filosofía Práctica de Alberto, bastaría con permanecer en los límites de la lectura y del tratado De natura boni comentarios elaborado por el Doctor universalis. A partir del horizonte de reflexión teológica, que predominó hasta el siglo XII, Alberto fue el primero en recibir, comentar e incorporar las tesis de Aristóteles, especialmente las referidas al nivel de la Filosofía Política, He dicho ya, en otros escritos, que curiosamente Alberto descubrió la intencionalidad política de la Ética de Aristóteles, aún sin haber leído la totalidad de sus libros éticos y políticos. No solo eso, sino que también meditó acerca de la traducción moral de una felicidad, que no debe detenerse en la pura contemplación. El lugar concreto donde esta novedosa posición puede reconstruirse es el libro X de los dos Comentarios a la Ética a Nicómaco (E-I y E-II) ${ }^{39}$. Más tarde, con Tomás de Aquino, esa misma idea se acuñaría en el axioma agere sequitur esse. Es como si Alberto, en virtud de su neo-platonismo,

${ }^{37}$...non quaeritur coniunctio ad veritatem per intellectum tantum, sed etiam per affectum et substantiam; et ideo non est intellectiva sed affectiva, quia intelectus ordinatur ad affectum ut ad finem ...Finis doctrinae istius determinatur ad TIT, I-9, ubi dicitur: Amplectentem eum qui secundum doctrinam est fidelem sermonem, ut potens sit exhortari in doctrina sana et revincere contradicentes. Finis autem scientiae dúplex est, scilicet ultimus et ordinatus ad illum. Qui a quibusdam terminus; finis autem citra illum est, ut boni fiamus (Albertus Magnus, 2015 pp. 14-56, 15-1).

${ }^{38}$ Respecto del concepto de intelecto adquirido, cabe destacar que el mismo fue recogido por Alberto Magno de la obra de Al-Farabi. Véase (Al-farabi, 1930, pp. 121-122). Alberto formula la misma referencia en la Summa Theologicae, p. 2-3. Hinc est, quod dicit Alfarabius in libro De Intellectu et Intelligibili, quod omnes philosophi in intellectu adepto divino radicem posuerunt immortalitatis animae. Respecto de la evidente relación que existe entre las doctrinas del intelecto adquirido y la del intelecto agente en el pensamiento árabe, recogida y modificada por Alberto Magno, cabe leer, además de las presentes reflexiones, los textos que sumariamente indico a continuación. Véase (Al-Farabi, 2008; 1985); (Mahdi, 2001, p. 229).

${ }^{39}$ Véase (Albertus Magnus, 1891, p. 2). 
hubiera podido detectar desde el comienzo, aquellos elementos platónicos, que permanecían potencialmente en la reflexión política de Aristóteles. Los mismos le permitieron amalgamar definitivamente la Ética y la Política con la Filosofía Primera y con la Teología Sobrenatural. La esfera en la que esa armónica unidad tiene lugar, es la del intelecto adquirido.

La exigencia de Alberto en punto a comenzar la reflexión moral y política a partir de la posesión del intellectus adeptus-intelecto adquirido ${ }^{40}$, además de excluir toda autonomía entre contemplación y obrar, indica claramente que el más elevado conocimiento de Dios que el hombre puede alcanzar, repotenciado por la Fe, la Revelación y los Signos que de Dios emanan, es el que se traduce en rigor como regulación normativa del obrar moral y político. Aquella scientia affectiva et glorificans, la scientia suprasubstantialis, está presente hora en el intellectus adeptus. Por tanto, es en esta perfección del intelecto humano que llamamos intelecto adquirido, donde se unen el Tiempo y la Eternidad. La configuración del intellectus adeptus es el resultado obtenido mediante la convergencia de las dos recapitulaciones del saber humano. Esa ciencia, cuyo objeto inicialmente no era otro que Dios, se torna luego scientia utens-ciencia útil-como lo es la Filosofía Política, teniendo como finalidad aquella completa transformación interior del hombre, que se torna visible por su obrar y que Alberto define como ut boni fiamus-para que seamos buenos- ${ }^{41}$. Esa scientia, vista en orden a las operaciones voluntarias, se traduce finalmente, en el nivel de una Filosofía Política, como scientia oboediens, desde que reconoce en las Sagradas Escrituras, en el Ordo Creationis y en el ordo naturae, su horizonte clarificador y justificador por antonomasia.

\section{Conclusiones}

Por último, cabe formular algunas breves conclusiones. Las unas de orden epistemológico y las otras de carácter filosófico-político. Desde el punto de vista epistemológico; sin duda la Filosofía Política de Alberto, como más tarde será el caso con la de su discípulo Tomás de Aquino, pertenece al todo de la Filosofía, recibiendo de las disciplinas filosóficas superiores, que son la Metafísica y la Teología Sobrenatural, sus límites. De la Metafísica recibe la fundamentación última del Bien Político en el bien como trascendental del Ser y de la Teología Sobrenatural, recibe su límite en el sentido que la verdad

${ }^{40}$ Véase (Anzulewicz, 2003, p. 169).

41 "Intellectus speculativus extendendo se fit practicus" (Albertus Magnus, 2008, pp. 295-372). Aun con mayor claridad, dice Alberto, "speculativus et practicus conveniunt in substantia et differunt in ratione. Et haec ratio est extensio a vero in bonum (Albertus magnus, 2008, pp. 485-491). Respecto del nivel epistemológico: (Albertus Magnus, 1987, pp. 392-55). 
política no puede ser contraria a la Verdad Sobrenatural y Revelada. Por su parte, esa Filosofía Política es la más importante de entre todos los modos del saber humano, pues su objeto es el bien más alto al que el hombre puede aspirar in via, esto es el Bien Común Político. Precisamente en el interior de esa totalidad de carácter onto-teológico es que el conocimiento de las grandes cuestiones políticas puede experimentar las dos recapitulaciones del saber humano. Si la primera recapitulación del saber humana fue propuesta por Aristóteles y por Platón, la segunda fue decididamente experimentada por Alberto y por Tomás a la luz de las tesis recibidas de Dionisio el Aeropagita.

El cuarto grado de abstracción que marcaba el fin del camino filosófico, para iniciar el camino rigurosamente teológico y el de la fe Sobrenatural, nos permitió poner en evidencia la cualitativa transformación del concepto platónico de virtus politica. El homo politicus de Alberto es claramente un homo religiosus. La prueba de la pertenencia de la Filosofía Política a la reflexión ofrecida por la Teología Sobrenatural y por la Metafísica la ofrece Alberto cuando en el punto de partida de su prólogo al Comentario a la Política de Aristóteles, postula su tesis acerca del intelecto adquirido, vale decir, de aquella perfección de nuestra inteligencia en cuyo interior se unen el Tiempo y la Eternidad. Por ese motivo, finalmente, la Filosofía Política del Doctor universalis, es una Filosofía abierta a la Trascendencia. La Filosofía Política de Alberto es, en definitiva, scientia utens et transcendens.

\section{Referencias bibliográficas}

Agustín de Hipona. (2015). Obras Completas de San Agustín [Tomo XIX]. Madrid,

España: Biblioteca de Autores Cristianos (BAC).

Albertus Magnus. (1891). Ethicorum (Libro X). París, Francia: Augusto Borgnet. Albertus Magnus. (1960). Metaphysica [Tomo XVI - Volumen I]. Munich, Alemania: Aschendorff Verlag.

Albertus Magnus. (1972). Super Dionysium de Divinis Nominibus [Tomo XXXVII

- Volumen I]. Munich, Alemania: Monasterii Westfalorum, Aschendorff.

Albertus Magnus. (1974). De natura boni [Tomo XXV-1]. Munich, Alemania: Ed. Colon, Ed. Aschendorff.

Albertus Magnus. (1975). Super ethica Commentum et Quaestiones [Tomo XIV -

Volumen I]. Munich, Alemania: Aschendorf-Münster.

Albertus Magnus. (1978a). Super Dionysii Mysticam Theologiam et Epistulas [Tomo XXXVII - Volumen II]. Munich, Alemania: Aschendorf- Münster.

Albertus Magnus. (1978b). Summa theologiae [Tomo XXXIV - 1]. Munich, Alemania: Aschendorf-Münster.

Alberto Magnus. (1987). Super ethica Commentum et Quaestiones (E-I). París,

Francia: Aschendorff. 
Albertus Magnus. (2008). De homine [Tomo XXVII - Volumen II]. Munich, Alemania: Aschendorff-Münster.

Albertus Magnus. (2015). Super I Librum Sententiarum. Distinctiones 1-3 [Tomo XXIX - Volumen I]. Munich, Alemania: Aschendorf-Münster.

Al-Farabi. (1930). De Intellectu et Intellecto. En E. Gilson. (Ed.), Les sources gréco-arabes de l'augustinisme avicennisant [Volumen 4] (pp. 115-126). París, Francia: Archives D'Histoire Doctrinale et Littéraire du Moyen Age.

Al-Farabi. (1985). On the perfect state. Oxford, Inglaterra: Great books of the Islamic World.

Al-Farabi. (2008). Libro de política. En R. Guerrero. (Ed.), Obras Filosóficas y Políticas (pp. 53-54). Madrid, España: Abu Nasr Al-Farabi.

Anzulewicz, H. (2003). Entwicklung und Stellung der Intellekstheorie im System des Albertus Magnus. Arhives d'Histoire Doctrinale et Littéraire du Moyen Age, 70, 165-218.

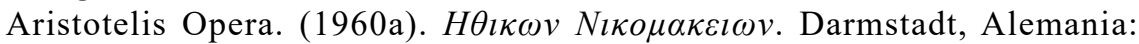
Wissenschaftliche Buchgesellschaft.

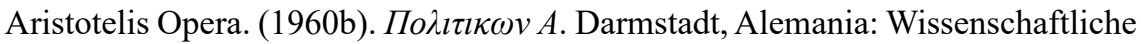
Buchgesellschaft.

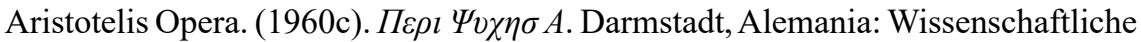
Buchgesellschaft.

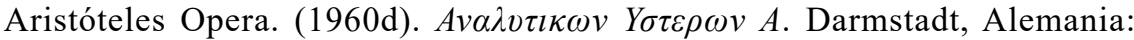

Wissenschaftliche Buchgesellschaft.

Benavidez, C. E. (2018). El cominciamento filosófico en Tomás de Aquino y Hegel según Cornelio Fabro. Praxis Filosófica, (46), 171-187. doi: 10.25100/ pfilosofica.v0i46.6166

Díaz, J. A. (2017). Pecado y Autonomía. Praxis Filosófica, (45), 259-283. doi: 10.25100/pfilosofica.v0i45.6061

Folghera, I. O. P. (1925). Saint Thomas et la prédication. En L. Theissling, Xenia Thomistica (pp. 583-595). Roma, Italia: Editorial Angelicum.

Fries, A. (1994). Albertus Magnus. Ausgewählte Texte. Darmstadt, Alemania: Wissenschaftliche Buchgesellschaft.

Geyer, B. (1967). Die Patristische und Scholastische Philosophie. En F. Überwegs, Grundriss der Geschichte der Philosophie (Vol. II). Darmstadt, Alemania: Wissenschaftliche Buchgesellschaft.

Granados, J. (3 de mayo de 2017). ¿Gueto, Masa o Minoría Creativa? [Mensaje en un blog]. Recuperado de https://www.almudi.org/articulos/11731-gueto-masao-minoria-creativa-la-fecundidad-sacramental

Hellmann, M. (2002). Apuleius im Mittelalter. En Lexikon des Mittelalters (Tomo I) (pp. 818-819). Stuttgart, Alemania: Deutscher Taschenbuch.

Höffe, O. (1996a). Praktische Philosophie. Das Modell des Aristoteles. Berlín, Alemania: Akademie Verlag.

Höffe, O. (1996b). Aristoteles. Munich, Alemania: C. H. Beck

Honnefelder, L. (2011). Albertus Magnus und der Ursprung der Universitätsidee: Die Begegnung der Wissenschaftskulturen im 13. Jahrhundert und die 
Entdeckung des Konzepts der Bildung durch Wissenschaft. Berlin, Alemania: Herausgegeben Von Ludger Honnerfelder.

Mahdi, M. (2001). Alfarabi and the Foundation of Islamic Political Philosophy. Chicago, EUA: The University of Chicago Press.

Müller, J. (2006). Der Einfluss der arabischen Intellektspekulation auf die Ethik des Albertus Magnus. En A. Speer y L. Wegner. (Eds.), Wissen über Grenzen. Arabisches Wissen und lateinisches MIttelaleter, [Tomo 33]. Berlín, Alemania: Miscelanea Mediaevalia.

Sancti Thomae Aquinatis. (1888). Summa Theologiae [Prima Pars, Q. 1, Art. VI]. Roma, Italia: Fundación Tomás de Aquino. Recuperado de http://www. corpusthomisticum.org/sth1001.html 
